\title{
IMIQUIMOD EN CREMA AL 5\% EN EL TRATAMIENTO DE VERRUGAS ANOGENITALES ASOCIADAS A VPH: EXPERIENCIA PRELIMINAR
}

\author{
Omar Nazzal N. 1, Eugenio Suárez P. 1, Marcia Venegas a, Raúl Larraguibel P. ${ }^{1}$, \\ Luciano Rojas F. ${ }^{1}$, Alessandro Bronda M. ${ }^{1}$, Miguel Saavedra C. ${ }^{1}$, Jessica Rojas U. ${ }^{1 b}$ \\ 1 Unidad de Ginecología Oncológica Servicio y Departamento de Obstetricia y Ginecología Hospital Clínico San Borja \\ Arriaran Facultad de Medicina Campus Centro Universidad de Chile
}

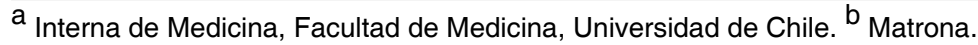

\section{RESUMEN}

Antecedentes: La infección persistente por VPH de alto riesgo se asocia con cáncer cervicouterino, encontrándose positividad en el $99,7 \%$ de los casos. Las verrugas anogenitales son una forma de presentación de la infección persistente. Objetivo: Investigar la eficacia y seguridad de imiquimod en crema al $5 \%$, en el tratamiento de las verrugas anogenitales en mujeres. Método: Estudio prospectivo no randomizado, en mujeres portadoras de verrugas anogenitales asociadas a VPH. El tratamiento consiste en la autoaplicación de imiquiomod en crema al $5 \%$ tres veces a la semana por hasta un período de 16 semanas. Se define como respuesta completa al tratamiento aquella en que desaparece completamente la lesión; por respuesta parcial la disminución de al menos un $50 \%$ del volumen inicial de la lesión. Resultados: Durante un período de 8 meses fueron ingresadas y tratadas 38 pacientes La duración del tratamiento varió entre 1 y 4 meses. El número promedio de aplicaciones fue de 28,7 . El $66 \%$ (25 casos) de las pacientes presentaron una respuesta completa, en tres casos no hubo respuesta, uno de ellos en una paciente inmunodeprimida, en 10 casos hubo respuestas parciales. En las pacientes con respuestas completas se necesitó un promedio de 28,7 aplicaciones hasta obtener el resultado. Conclusiones: Imiquimod es un fármaco con buena efectividad y seguridad en el tratamiento de las verrugas anogenitales asociadas a VPH, en tratamiento de tres veces por semana durante mínimo de 10 semanas.

\section{PALABRAS CLAVES: Virus papiloma humano, verrugas anogenitales, imiquimod, inmunomodulación, auto aplicación}

\section{SUMMARY}

Background: The chronic infection by high-risk HPV serotypes is strongly associated with cervical cancer, detection of HPV on tissue sample has been reported $99.7 \%$ of cases. The anal-genital warts represent the clinical manifestation of the persistent infection by this virus. Objectives: To investigate the efficacy and safety of Imiquimod cream 5\% concentration, in the treatment of anal-genital warts in women. Methods: A nonrandomized prospective study in women diagnosed with anal-genital warts HPV associated. The treatment consists in self-application of imiquimod cream $5 \%$, three times a week for a period of 16 weeks or more. We define complete response to treatment in those patients where the lesion disappeared completely, and partial response to those where the lesion declined at least $50 \%$ of its initial volume. Results: Over a period 
of 8 months were admitted to the study and treated a total of 38 patients. Treatment duration varied between 1 and 4 months. The average number of applications was 28.7 times. $66 \%$ (25 cases) of the patients had a complete response, in three cases there was no response, one of them was an immunodepressed patients, in 10 cases there was partial response. The patients with complete response needed an average of 28.7 applications to get that result. Conclusions: Imiquimod is a drug with high effectiveness and safety in the treatment of anal-genital warts HPV associated, in a three times per week and at least 10 weeks total duration treatment.

\section{KEY WORDS: Human papillomavirus, anal-genital warts, imiquimod, immunomodulation, self-aplication}

\section{INTRODUCCIÓN}

La incidencia de infecciones de transmisión sexual son un problema creciente de salud pública en Chile y el mundo. Dentro de ellas destacan las asociadas al virus Papiloma Humano (VPH). Los condilomas acuminados son la manifestación clínica de los genotipos 6 y 11 de VPH, responsables del $90 \%$ de las verrugas genitales en mujeres y hombres. Se estima que afectan al $1 \%$ de las mujeres sexualmente activas entre 15 y 49 años (1). La infección persistente por VPH de alto riesgo se asocia fuertemente con cáncer cervicouterino, encontrándose positividad para ellos en el 99,7\% de los casos (2). Si bien la morbilidad asociada a la presencia de condilomas acuminados no es significativa, el estrés psicológico que significa su presencia hace imprescindible su tratamiento en forma eficaz (3).

Múltiples terapias se describen en el tratamiento de las verrugas genitales, a saber, ácido tricloroacético, podofilina, 5 fluoruracilo tópico, diatermocoagulación, láser, exéresis quirúrgica entre otros. En el último tiempo se ha comenzando a difundir el tratamiento en base a crema de imiquimod al $5 \%$ en forma de auto - aplicación por el paciente. Imiquimod es una imidazoquinolina de bajo peso molecular, que actúa como un modulador de la respuesta inmune, induciendo citokinas que promueven una respuesta inmunológica mediada por linfocitos Thelper o una respuesta inmune de tipo celular, fundamentalmente a nivel local (3).

El objetivo de este estudio es investigar la eficacia y seguridad del imiquimod (Tocasol ${ }^{\circledR}$ en crema al $5 \%$ ), en el tratamiento de las verrugas ano genitales en mujeres, comercializado por el Laboratorio Gynopharm.

\section{MATERIAL Y MÉTODO}

Estudio prospectivo no randomizado, en mujeres portadoras de verrugas anogenitales asociadas a VPH, que consultan en la Unidad de Patología Cervical y/o Policlínico de ETS del CDT Hospital Clínico San Borja-Arriarán, Campus Centro, Facultad de Medicina Universidad de Chile. El tratamiento consistió en la autoaplicación de imiquiomod en crema al $5 \%$ tres veces a la semana por hasta un período de 16 semanas. En aquellas pacientes que eliminaron las verrugas, se efectuó seguimiento hasta los 6 meses de finalizado el tratamiento. Las pacientes que recurren pueden ser retratadas con imiquimod mediante segunda intención terapéutica, por un periodo adicional de 16 semanas. En casos de respuestas parciales se puede extender el período de tratamiento. Todas las pacientes fueron examinadas colposcópicamente antes del inicio del tratamiento y cada 4 semanas por el mismo examinador consignándose en cada visita las reacciones adversas. En la primera consulta se dio a las pacientes una charla educativa, así como adiestramiento para la autoaplicación de la crema, entregándoseles además un folleto recordatorio de la autoaplicación. Cada paciente firmó un consentimiento informado antes del inicio del tratamiento. En cada control, el examinador o la matrona de UPC realizó un reforzamiento de la educación, y control de técnica auto aplicación. Para cada caso se asignó una ficha de ingreso al estudio, la cual consignaba datos de identificación, patología asociada, factores de riesgo relacionados con verrugas genitales, evaluación inicial, características de cada control efectuado, y evaluación final de respuesta.

Una respuesta completa se definió como la desaparición de la lesión (ausencia de verrugas). Por respuesta parcial, entenderemos la disminución de al menos un $50 \%$ del volumen inicial en la lesión, utilizando regla de medición, aplicada en el diámetro mayor de esta y evaluación de imagen digital.

Se efectuó un registro de imágenes para cada caso, antes de inicio del tratamiento, medición en cada uno de los controles mencionados previamente, así como análisis por un panel de ginecólogos oncólogos al finalizar el tratamiento. Para cada 
caso se efectuó registro colpofotográfico utilizando cámara video digital asociada a colposcopio, o captura y almacenamiento de imágenes a través de software Olympus® (Figura 1).

\section{RESULTADOS}

Durante un período de 8 meses fueron ingresadas al estudio y tratadas un total de 38 pacientes con un promedio de edad de 26,7 años (rango: 1549 años). De ellas, 20 presentaban lesiones múltiples $(52,6 \%)$ y $18(47,4 \%)$ lesiones únicas. El tamaño promedio de las lesiones fue de $13 \mathrm{~mm}$ (rango: 4-30 mm). La duración del tratamiento varió entre 1 y 4 meses con un promedio de 2,4 meses. El número promedio de aplicaciones fue de 28,7 (rango: 12-36).

El $66 \%$ (25 casos) de las pacientes, presentaron una respuesta completa, en tres casos no hubo respuesta, uno de ellos en una paciente inmunodeprimida portadora de un transplante renal; en 10 casos hubo respuestas parciales, siendo tres de ellos lesiones en muslo lejanas al área genital. En las pacientes con respuestas completas se necesitó un promedio de 28,7 aplicaciones hasta obtener el resultado (2,4 meses) (Figuras 2 y 3). En cuatro casos se presentó prurito en la zona de aplicación, el cual remitió espontáneamente. Solo una paciente presentó una reacción adversa moderada al tratamiento, con lesión ulcerada en vulva que fue tratada con interrupción del tratamiento y medidas locales con buena repuesta, reiniciándose el tratamiento con respuesta completa.

\section{DISCUSIÓN}

Imiquimod es un inmunomodulador que actúa inhibiendo la replicación del VPH permitiendo la regresión de las verrugas anogenitales. Su acción se basa en la combinación de la activación de la inmunidad local por medio del interferón alfa y la activación de la inmunidad adquirida estimulando una respuesta inmune $\mathrm{T}$ dependiente $(3,4,5)$.

En este estudio se comprueba que imiquimod es relativamente eficaz y seguro, consecuente con diversos estudios que se han presentado, y que llegan a la misma conclusión. En un estudio de Garland y cols (6), comprobaron que imiquimod tenía una efectividad del $65 \%$ de respuesta completa en el tratamiento de las verrugas anogenitales produ-

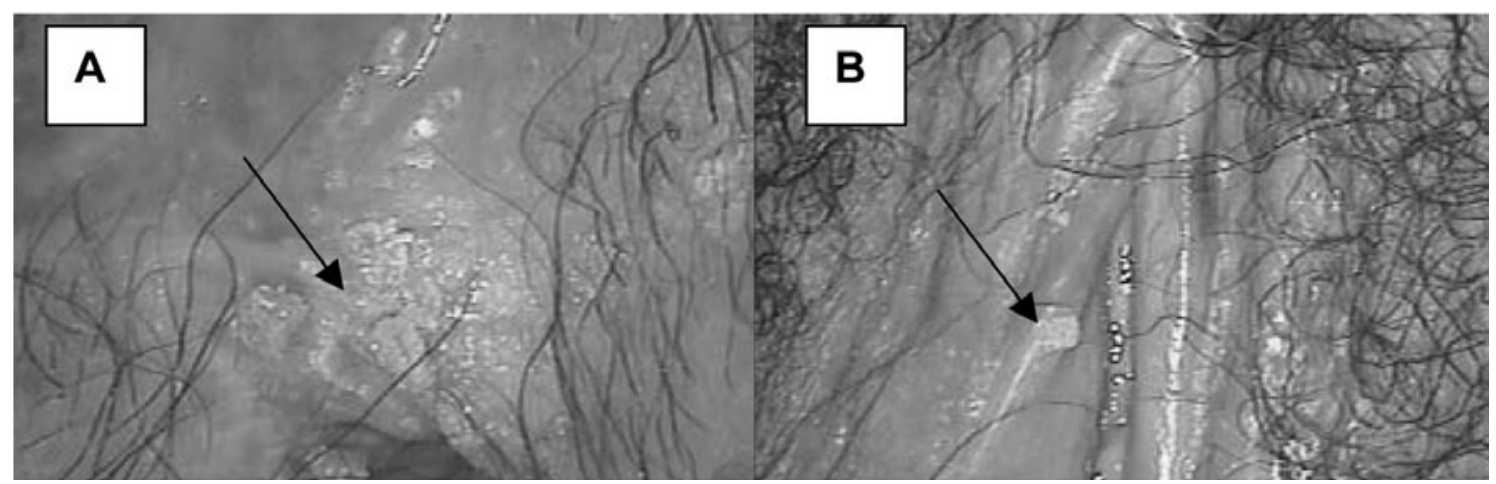

Figura 1 A-B. A: condiloma perianal; B: condilomas vulvares.

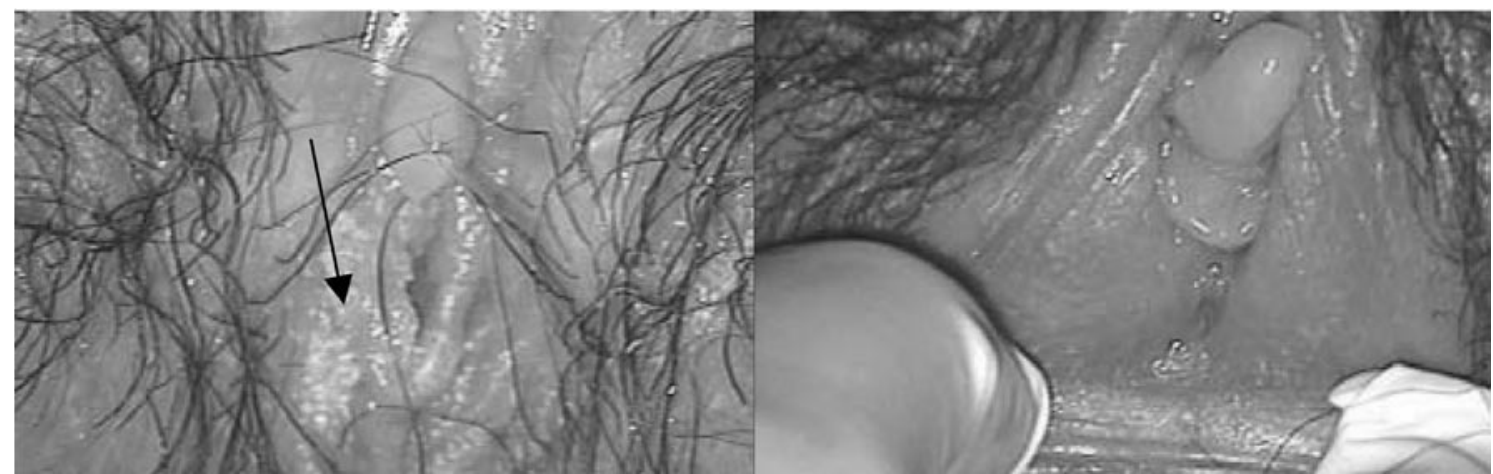

Figura 2. Condilomas vulvares y cuatro semanas después. 


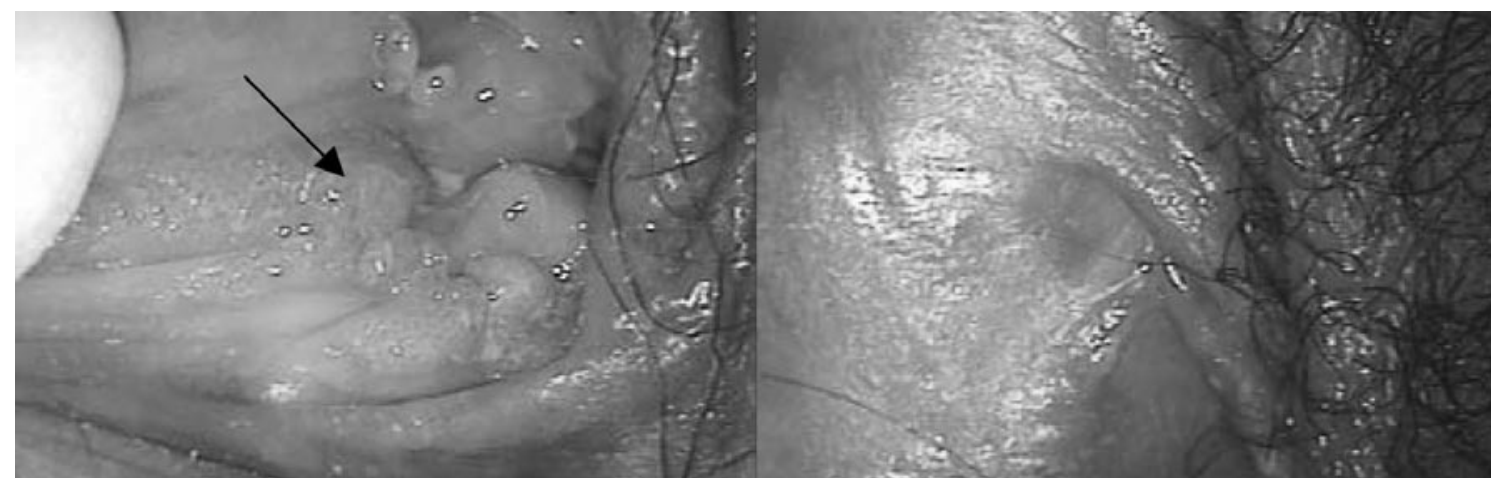

Figura 3. Condilomas vulvares y siete semanas después.

cidas por VPH, cuando se utilizaba tres veces por semana por un mínimo de 16 semanas. La importancia de este estudio radica en el tamaño muestral de 943 pacientes. En nuestro estudio se obtuvo una efectividad similar (66\%) cuando se realizaba la terapia con aplicación de tres veces por semana por aproximadamente 10 semanas. En diversos estudios se ha comparado la efectividad de imiquimod en relación a otras terapias ampliamente utilizadas como podofilina, y en todos se concluye que imiquimod es el tratamiento inicial más costo-efectivo que actualmente hay disponible, considerando el porcentaje de curación total, porcentaje de recidivas y costo de la terapia, junto con el hecho de ser un tratamiento que se aplica en casa y por la misma paciente, disminuyendo el estrés psicológico de la situación $(1,4,7,8,9)$.

En un estudio realizado por Schöfer y cols $(10,11)$, se comparó la efectividad de la terapia de verrugas anogenitales con imiquimod como monoterapia, versus imiquimod asociado a terapia ablativa, versus terapia ablativa sola. Concluyeron que imiquimod $5 \%$ en crema, como monoterapia o en combinación con terapia ablativo, fue más efectiva que la terapia ablativa sola, en reducir las recurrencias de las verrugas anogenitales tratadas con respuesta completa. De esta forma todo apunta a que la terapia con imiquimod crema al 5\% parece ser una muy buena alternativa para tratar las verrugas anogenitales, sobre todo como terapia inicial. Lo más razonable sería entonces utilizarla como primera opción evaluando la respuesta y la aparición de recidivas, y en caso que no se logre una remisión completa probar con otras terapias como la ablativa.

\section{CONCLUSIONES}

Imiquimod se constituye como un fármaco con buena efectividad y seguridad en el tratamiento de las verrugas anogenitales asociadas a VPH. Con un nuevo enfoque terapéutico basado en la inmunomodulación. Posee un favorable porcentaje de respuesta completa en aplicaciones de tres veces por semana por más de 10 semanas. La facilidad de aplicación, como tratamiento en casa y por la misma paciente, es un hecho importante cuando se compara con otro tipo de terapias. Podría resultar una muy buena alternativa como tratamiento inicial de las verrugas anogenitales, llegando a terapias más agresivas solo en los casos en que la respuesta no fue total.

\section{BIBLIOGRAFÍA}

1. Sauder DN, Skinner RB, Fox TL, Owens ML. Topical imiquimod $5 \%$ cream as an effective treatment for external genital and perianal warts in different patient populations. Sex Transm Dis 2003;30(2):124-8.

2. Walboomers JM, Jacobs MV, Manos MM, Bosch FX, Kummer JA, Shah KV, Snijders PJ, Peto J, Meijer CJ, Munoz N. J Pathol 1999;189:12-9.

3. Hober D, Ajram L, Chehadeh W, Lazrek M, Goffard A, Dewilde A, Wattré P. Mechanisms of imiquimod indirect antiviral activity. Ann Biol Clin (Paris) 2005;63(2):15563.

4. Lafuma A, Monsonego J, Moyal-Barracco M, Pribil C. A model-based comparison of cost effectiveness of imiquimod versus podophyllotoxin for the treatment of external anogenital warts in France. Ann Dermatol Venereol 2003;130(8-9 Pt 1):731-6.

5. Senatori R, Dionisi B, Lippa P, Inghirami P. Topical imiquimod cream in the treatment of external anogenital warts: personal experience. Minerva Ginecol 2003;55(6):541-6.

6. Garland SM, Sellors JW, Wikstrom A, Petersen CS, Aranda C, Aractingi S, Maw RD; Imiquimod Study Group. Imiquimod $5 \%$ cream is a safe and effective self-applied treatment for anogenital warts--results of an open-label, multicentre Phase IIIB trial. Int J STD AIDS 2001;12(11):722-9. 
7. Vilata JJ, Badia X; ESCCRIM group. Effectiveness, satisfaction and compliance with imiquimod in the treatment of external anogenital warts. Int J STD AIDS 2003;14(1):11-7.

8. Edwards L, Ferenczy A, Eron L, Baker D, Owens ML, Fox TL, Hougham AJ, Schmitt KA. Self-administered topical 5\% imiquimod cream for external anogenital warts. HPV Study Group. Human PapillomaVirus. Arch Dermatol 1998;134(1):25-30.

9. Perry CM, Lamb HM. Topical imiquimod: a review of its use in genital warts. Drugs 1999;58(2):375-90.

10. Schöfer H, Van Ophoven A, Henke U, Lenz T, Eul A. Randomized, comparative trial on the sustained efficacy of topical imiquimod $5 \%$ cream versus conventional ablative methods in external anogenital warts. Eur J Dermatol 2006;16(6):642-8

11. Schöfer $H$. Evaluation of imiquimod for the therapy of external genital and anal warts in comparison with destructive therapies. Br J Dermatol 2007;157(Suppl 2):52-5. 